\title{
REVISIA INVESTIGACIÓN EN INSTITUCIONES DE EDUCACIÓN SUPERIOR COMO PROCESO SUSTANTIVO INTEGRADO: SU RETO ENDÓGENO
}

\author{
RESEARCH IN HIGHER EDUCATION INSTITUTIONS AS A \\ SUBSTANTIVE DUE PROCESS: THE ENDOGENOUS CHALLENGE
}

\author{
Eneida Catalina Matos Hernández ${ }^{1}$ \\ Lorna Cruz Rizo' \\ 1 Universidad de Guayaquil, Ecuador. ematoshernandez@yahoo.es
}

\section{RESUMEN}

A partir de la amplia demanda social actual a las universidades de resultados de investigación emergentes, en su relación con la fragmentación de los procesos sustantivos universitarios que ha estado limitando el proceso de producción de conocimiento científico, se presenta un estudio sobre el proceso investigativo en Instituciones de Educación Superior, desde su integración con los restantes procesos sustantivos universitarios: la formación académica y la gestión social del conocimiento. A través de la utilización de la sistematización de experiencias como eje articulador de la metodología investigativa utilizada, en coherencia con la revisión documental de páginas web de universidades latinoamericanas, se revelan las inconsistencias teórico-prácticas en la producción de conocimiento científico, desde las esencialidades del contexto cultural universitario propio, por lo que se marcan inconsistencias en los procesos endógenos, a partir de lo cual se propone una lógica procesual de la investigación endógena. Dicha lógica, por su nivel de esencialidad, es aplicable a una diversidad de contextos universitarios, siempre que se necesite potenciar la investigación endógena, integrada al resto de los procesos sustantivos.

Palabras Clave: investigación, procesos sustantivos, conocimiento científico, sistematización, endogenización.

\begin{abstract}
From the wide current social demand for emerging research results to universities, in its relation to the fragmentation of university substantive due processes that has been interfering in the scientific knowledge process of production, an study of the research process in Higher Education Institutions is presented, from its integration with the rest of the university substantive due processes: academic formation and the social management of knowledge. Through the systematization of experiences as the joint axis of the research methodology used, in a coherent relation to the revision of web pages form Latin American universities, theoretical and practical inconsistencies were revealed in the scientific knowledge process production, from the essences of the own university cultural context, that is why a fissure is shown in the endogenous processes, from which a procedural rationale of the endogenous research is proposed. Said rationale, due to its essentiality level, is applicable to a diversity of university contexts, as far as they need to potentiate the endogenous research integrating due processes.
\end{abstract}

Keywords: research, substantive due processes, scientific knowledge, systematization, endogenization.

Recibido: 30/11/2017; $\quad$ Aceptado: 07/12/2017; $\quad$ Publicado: 19/12/2017

\section{Introducción}

La investigación está llamada a ser el eje articulador de la vida institucional en las universidades. A pesar del vertiginoso desarrollo actual de la ciencia, la tecnología y las comunicaciones, el ritmo de producción de nuevos conocimientos y de comunicación de información actualizada, aún no logra satisfacer, en tiempo real, las demandas sociales. 
El desarrollo de las sociedades sigue estando mediado por una multitud de influencias económicas, políticas, psicológicas, mediáticas, comunicacionales, étnicas, religiosas, territoriales, en fin de diversa naturaleza, que constantemente están generando nuevas situaciones disfuncionales, desde una diversidad de espacios culturales, entendiendo aquí la cultura como ese complejo entramado de sistemas de relaciones entre categorías. De lo cual se asume que este desarrollo social deberá fundamentarse en la cultura universitaria, que ha sido asumida por estas autoras como: "el sistema de significados formativos contextuales en el que se dinamizan los contenidos profesionales en relación con las demandas sociales a través de las funciones sustantivas universitarias." (Matos \& Cruz, 2016, p. 3)

La integración de las funciones sustantivas universitarias, por tanto, asume un rol de liderazgo institucional, en el cual los retos a la investigación científica están siendo cada vez más complejos y diversos, en tanto ya no es posible dar respuesta a los problemas sociales desde una apreciación parcial de un investigador o de una ciencia particular. Las universidades deben retomar su rol histórico de productoras de conocimiento científico de excelencia, como manifestación de su sentido de compromiso social. Siendo así, las universidades están siendo llamadas a asumir una postura integradora de sus procesos investigativos, como concreción praxiológica de los procesos de formación académica y de gestión social del conocimiento, pues solo así será posible formar profesionales que sean capaces de generar soluciones a los problemas sociales en el menor tiempo posible, con pertinencia e impacto, además de lograr una optimización de los recursos utilizados para lograr dichas soluciones.

En dicho orden de aspiración de una investigación de calidad, teóricamente han quedado establecidas las pautas para una investigación universitaria compleja e interrelacionada, en la que el nuevo conocimiento obtenido vaya más allá de la visión limitada que las partes de un objeto, proceso o fenómeno pueden ofrecer de manera fragmentada; logrando una integración del todo, que como cualidad superior, logre expresar lo más cercanamente posible, aquellos rasgos o cualidades que tipifican el desarrollo sostenible. Estas pautas han llevado a enfrentar la complejidad como la única alternativa posible para una investigación científica que provea de soluciones emergentes a los problemas sociales, a través de la contextualización e integración de saberes, la complejización del conocimiento y la democracia cognitiva. (Morín, 2001)

Siendo así, las Instituciones de Educación Superior (IES) han venido marcando la relevancia de los procesos investigativos que se desarrollan de manera intra (al interior de la universidad, entre sus sujetos e instancias de formación) e interinstitucional (entre la universidad y el resto de las instituciones sociales) al reconocer a la investigación como uno de sus procesos sustantivos, es decir como un proceso del cual depende la existencia misma de la universidad como institución social.

Sin embargo, las universidades, como generalidad, han estado asumiendo estructuras y procesos que han sido funcionales en otros contextos y que, no siempre responden a las esencialidades culturales, mayormente de talento humano y de cualidades de las ciencias que se investigan, de sus propios contextos de actuación investigativa. Lo anterior es evidente en la similitud de las estructuras, planes, programas, proyectos que internacionalmente se aplican, aun cuando dicho sistema no haya demostrado resultados loables en determinados espacios.

Se considera aquí que la implementación de modelos exógenos de investigación, sin la necesaria mediación y contextualización, puede estar influyendo negativamente en la prontitud y pertinencia de los resultados de la investigación institucional, sobre todo en cuanto a la integración coherente de la gestión investigativa con la formación académica y la gestión social del conocimiento. Es por ello, que emerge la necesidad de gestionar la investigación endógena desde su integración con la formación académica y la gestión social del conocimiento, lo que se revela como el problema científico de esta investigación.

Por ende, el presente artículo, a través de una sistematización praxiológica, caracteriza a la investigación como proceso sustantivo a nivel universitario, para precisar los rasgos que actualmente tipifican su organización sistémica y funcionamiento. Al respecto, es necesario identificar el nivel de integración de la investigación con el resto de las funciones sustantivas, en tanto de ello depende la coherencia funcional de la vida institucional. De igual modo, se hace necesario distinguir la existencia de modelos de investigación que sean expresión de las cualidades identitarias de las universidades, como procesos endógenos de desarrollo investigativo institucional. Finalmente, se presenta una lógica procesual 
de la investigación endógena, que demanda una integración de esta función sustantiva con el resto de los espacios existenciales universitarios.

La investigación como proceso sustantivo se asume aquí como el proceso de producción, aplicación y comunicación de nuevos conocimientos científicos como respuesta a las demandas sociales, en estrecha interrelación con los procesos de formación académica y de gestión social del conocimiento. Esta función sustantiva: "comprende todas las actividades asociadas con el trabajo desarrollado por los investigadores agrupados en organismos colegiados de investigación." (Velázquez \& Maldonado 2006, p.160)

Al respecto, Vergara-Quintero, MC., Et. Al., (2016) han planteado que:

La Investigación es una de las funciones sustantivas de la universidad, cuyo propósito es contribuir en la consolidación de una cultura institucional basada en la generación, apropiación y difusión del conocimiento científico, la innovación y el aprendizaje constante en la comunidad académica, para hacer de la investigación un hábito permanente. La investigación fundamenta el proceso de formación académica y promueve la educación de alta calidad; por tanto, se constituye en el eje de las transformaciones curriculares y de modernización de la Universidad. (p.72)

Siendo así, se reconoce aquí que, de manera cíclica, en esta función sustantiva se retroalimenta la formación académica, logrando que los actores universitarios generen productos y servicios tales como informaciones, capacitaciones, asesorías, dispositivos, softwares, consultorías, u otras, como resultado de la implementación de los conocimientos generados en la función académica y que se ofrecen a las instituciones sociales, empresas, organismos $u$ otras en la función de gestión social del conocimiento, lo cual a su vez genera una profundización en la solidez de los conocimientos gestionados en la academia, al

\footnotetext{
1 Universidad Nacional Autónoma de México, Universidad de Chile, Universidad Nacional de La Plata, Universidad de Puerto Rico, Universidad Nacional de Córdova, Argentina; Universidad Autónoma de Santa Ana, El Salvador; Universidad del Valle de Guatemala; Universidad Pedagógica Mariscal Sucre, Bolivia; Universidad de las Regiones Autónomas de la Costa Caribe Nicaragüense; Universidad San Pedro Perú.
}

2 Universidad Benemérita de Puebla, México (2005), Universidad Libre de Santa Fé de Bogotá, Colombia (2007, 2008, 2009, 2010, 2011); Universidad Nacional de las corroborar su valor de uso social, productivo o de servicios.

\section{Objetivo}

El objetivo general está en sistematizar el proceso de gestión de la investigación, como función sustantiva de las Instituciones de la Educación Superior, a partir de su integración con la formación académica y la gestión social del conocimiento.

\section{Materiales y Métodos}

Se utiliza en esta investigación, como métodos de investigación teórico, el análisis- síntesis y la inducción- deducción en interrelación con un enfoque sistémico que partió de la revisión documental de las páginas web de $d i e z^{1}$ universidades latinoamericanas, en las cuáles se realizó un análisis de los documentos que orientan su investigación como proceso sustantivo. Las diez universidades que se incluyen en este artículo son aquellas en las que se encontró alguna información representativa de la gestión de investigación institucional, pues en el resto de las páginas web revisadas no existía ninguna información al respecto.

De igual modo, el presente estudio se sustenta en la sistematización de experiencia de los investigadores involucrados, los cuales cuentan en su totalidad con más de veinte años de experiencia en el ejercicio de la docencia y la investigación científica en varias universidades latinoamericanas ${ }^{2}$

Se asume la sistematización como proceso que implica una dinámica de integración y generalización de saberes, (Celeiro, A., 2012) que no por ser experienciales pueden considerarse totalmente empíricos, pues han sido interpretados a través de indicadores de análisis, por lo que se cuenta con un registro de información devenida nuevo conocimiento científico, lo que implica que se ha desarrollado un proceso investigativo de naturaleza

Fuerzas Armadas (UNEFA) de Venezuela, en sus núcleos de Caracas y Cumaná (2005, 2006, 2007, 2008, 2009, 2010, 2011), Universidad de Oriente, Cuba (desde 1991 hasta el 2012), Universidad Estatal de Bolívar, Guaranda, Ecuador (2009, 2010, 2011), Universidad Tecnológica San Antonio de Machala, Ecuador (2012), Universidad Técnica de Machala, Ecuador (2013, 2014), Universidad Metropolitana, en su sede de Machala, Ecuador $(2013,2014)$, Universidad Laica Vicente Rocafuerte de Guayaquil (2014) y la Universidad de Guayaquil, Ecuador (2014, 2015, 2016, 2017). 
praxiológica, en el que se han creado nuevos conocimientos científicos, desde la misma práctica profesional, que a su vez se ha constituido en un espacio investigativo dinámico.

De acuerdo con los estudios de Jara Holliday $(\mathrm{s} / \mathrm{f})$ :

La sistematización es aquella interpretación crítica de una o varias experiencias que, a partir de su ordenamiento y reconstrucción, descubre o explicita la lógica del proceso vivido en ellas: los diversos factores que intervinieron, cómo se relacionaron entre sí y por qué lo hicieron de ese modo. La Sistematización de Experiencias produce conocimientos y aprendizajes significativos que posibilitan apropiarse de los sentidos de las experiencias, comprenderlas teóricamente y orientarlas hacia el futuro con una perspectiva transformadora. (p.4)

La sistematización de experiencias, por ende, como eje articulador de la investigación realizada, ha sintetizado información teóricopráctica a través de su ordenamiento y reconstrucción teórica, lo que permite enriquecer dicho proceso.

Se analiza aquí, entonces, el proceso de investigación científica en las IES estudiadas, especialmente en cuanto a la endogenización y la integración de sus procesos sustantivos, como criterios esenciales de la investigación realizada, que han guiado el análisis y la nueva construcción de conocimientos realizada.

\section{Resultados}

El estudio realizado partió de los siguientes criterios con sus respectivos indicadores:

Criterio 1: Estructura organizacional de la investigación científica

$>$ Estructuras coincidentes: Se consideran estructuras coincidentes aquellas que evidencian una organización en instancias colegiadas institucionales y que oscilan en un rango entre un 90 y $100 \%$ de coincidencia.

$>$ Estructuras parecidas: Se consideran estructuras parecidas aquellas que evidencian una organización en instancias colegiadas institucionales y que oscilan en un rango entre un 70 y $89.9 \%$ de coincidencia

Dstructuras diferentes: Se consideran estructuras diferentes aquellas que oscilan en un rango menor de $69.9 \%$.
Criterio 2: Gestión de la investigación científica institucional

> Gestión endógena: Se considera gestión endógena aquella que evidencia la existencia de documentos normativos, orientadores, que potencian la construcción del conocimiento científico a partir de las características propias de su talento humano y recursos materiales.

> Gestión mixta: Se considera gestión mixta aquella que imbrica características contextuales y universales en la gestión institucional de la construcción del conocimiento científico.

> Gestión exógena: Se considera gestión exógena aquella que evidencia la existencia de documentos normativos que potencian la construcción del conocimiento científico a partir de las características provenientes de otros contextos, diferentes del propio.

Criterio 3: Integración de la investigación con la formación académica y la gestión social del conocimiento.

$>$ Integradas: Se considera integrada aquella gestión de la investigación científica que evidencia documentos que integran espacios y objetivos compartidos con la formación académica y la gestión social del conocimiento.

$>$ De apoyo: Se considera de apoyo aquella gestión que evidencia documentos, en los cuales alguna función sustantiva colabora en el cumplimiento de objetivos de otra función sin llegar a considerarlos como metas propias.

> Fragmentadas: Se considera fragmentada aquella gestión de la investigación científica que evidencia documentos que no integran espacios y objetivos compartidos con la formación académica y la gestión social del conocimiento.

Las técnicas utilizadas para la compilación de datos fueron:

$>$ En el caso del primer criterio, se utiliza la escala gráfica de medición de datos donde se colocan valores opuestos, revelando una proporción continua para marcar el punto más cercano a la valoración realizada por los investigadores.

$>$ En el caso del segundo y tercer criterio, se utiliza la escala del diferencial semántico que contiene tres características a calificar y existen valores opuestos en ambos extremos.

Con respecto al primer criterio, los resultados alcanzados en la aplicación de la escala gráfica de medición de datos, evidencian que la 
generalidad de las Instituciones de Educación Superior estudiadas presenta una estructura organizacional mayormente coincidente. (Figura 1).

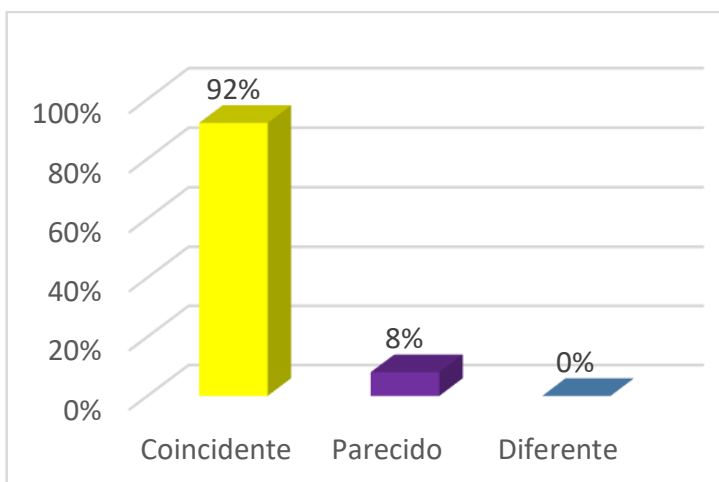

Figura 1.- Criterio 1: Estructura organizacional de la investigación científica.

En relación a la gestión de la investigación científica institucional analizada a través de la escala del diferencial semántico, se consideró la existencia suficiente 0 insuficiente de procesos institucionales endógenos, mixtos 0 exógenos, obteniéndose como resultado una insuficiente gestión endógena (Figura 2).

Este tipo de gestión se valoró teniendo en cuenta la correspondencia entre aspectos relacionados con la identidad contextual de la institución universitaria, y los indicadores establecidos para la producción científica. El primero referido a la cultura que incluye aspectos tales como la historicidad universitaria, formación en investigación de los actores y gestores universitarios, economía de la región, las costumbres, tradiciones, saberes y el segundo referido a: cantidad de publicaciones indexadas en determinadas bases de datos (regionales o internacionales), integración a redes de conocimiento, programas y proyectos, según los modos de producción del conocimiento.

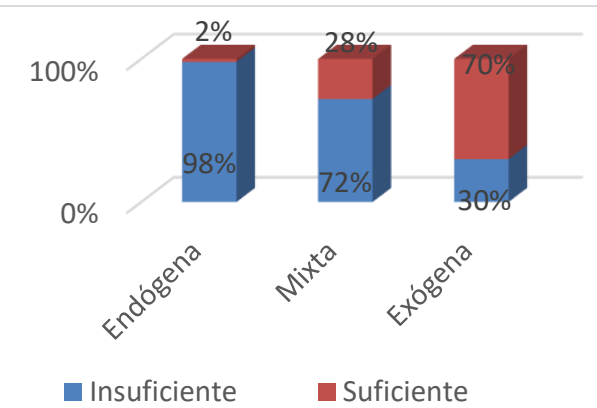

Figura 2.- Criterio 2: Gestión de la investigación científica institucional

Con respecto al criterio 3 referido a la integración de la investigación con la formación académica y la gestión social del conocimiento
(Gráfico 3), igualmente se utilizó la escala del diferencial semántico, valorándose la existencia de procesos de tres tipos: integrados, de apoyo o fragmentados. Se reveló la existencia de procesos mayoritariamente de apoyo y fragmentados.

Lo anterior se afirma en tanto la mayoría de los documentos revisados mostraron la existencia de procesos propios de cada una de las funciones, sin los necesarios vínculos o nexos integradores que se consideraron en esta investigación, los cuales deben quedar establecidos desde los documentos normativos $\mathrm{u}$ orientadores de la vida universitaria y concretados en la investigación institucional a través de objetivos, metas institucionales comunes, canales de comunicación, espacios de integración, Planes de Carrera o diseños curriculares, programas de asignaturas, prácticas pre-profesionales y programas comunitarios.

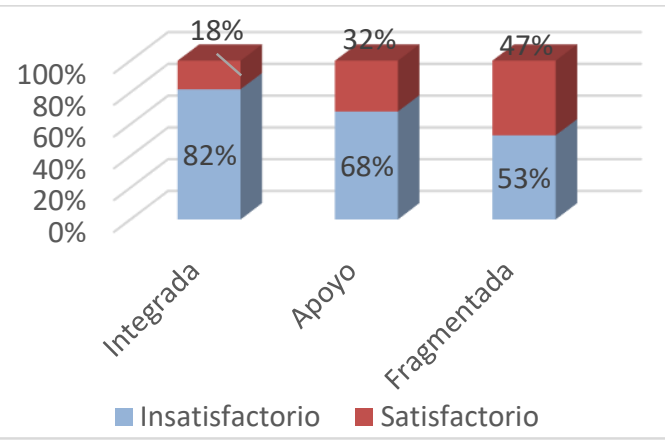

Figura 3.- Criterio 3: Integración de la investigación con la formación académica y la gestión social del conocimiento

\section{Discusión}

A partir del análisis de los resultados obtenidos en la sistematización praxiológica realizada en función de los documentos normativos internacionales, regionales y nacionales existentes respecto a la educación, la investigación y el desarrollo de la ciencia y la tecnología y los datos registrados por los investigadores participantes durante su desempeño profesional en las universidades referidas, es posible afirmar que dichas universidades, organizan su investigación de manera muy similar o coincidente. Esta organización de la gestión investigativa institucional parte de alguna instancia institucional, directamente subordinada al rectorado que, aunque con funciones similares, recibe diferentes denominaciones, entre ellas: Secretaría de Investigación y Desarrollo, Vicerrectorado de Investigación, Vicerrectoría de investigación y Desarrollo, Secretaría de Ciencia y Técnica, Secretaría de Ciencia y Tecnología, Dirección de Investigación y 
Producción de Conocimientos y Vicepresidencia de Investigación e Innovación, entre otras.

Esta instancia universitaria genera procesos de apoyo a los procesos investigativos institucionales, basándose en diferentes documentos tales como estrategias, reglamentos, planes, políticas, agendas y programas de investigación, entre otros, lo que a su vez implica la consecución de proyectos I+D+i con la consiguiente consideración de los recursos materiales y el talento humano necesario para impulsar la investigación institucional, desde la diversidad de facultades y carreras, con el objetivo de incrementar la cantidad y calidad de los resultados que se obtienen, en función del desarrollo socioeconómico.

En los últimos años, la gestión de la investigación universitaria ha incrementado sus vínculos con el sector productivo y empresarial, así como con las instituciones y áreas sociales que generan el desarrollo humano, por lo que a través de la función sustantiva de gestión social del conocimiento, se ha impulsado la creación de convenios con los actores y sectores del desarrollo local y el trabajo en red entre investigadores.

En este orden, desde la jerarquía institucional, la gestión de la investigación se organiza a partir de la existencia de directrices de investigación, relacionadas con los problemas más emergentes a los que la investigación institucional está llamada a dar respuesta científica (que pueden recibir la denominación de líneas de investigación, dominios u otros) como ejes temáticos que articulan un sistema de tareas y acciones de investigación, de un grupo o varios grupos de investigadores. Estas directrices temáticas constituyen un enfoque investigativo que debe aglutinar todo el sistema institucional, a través de la integración de sus funciones sustantivas y que han de impulsar de manera transdisciplinar el desarrollo de investigaciones. (Mintzbery y Water, 1985; Briceño y Chapín, 1988)

Al respecto, Barrios (1990) plantea que: "La línea de investigación es considerada como: el eje ordenador de la actividad de investigación que posee una base racional y que permite la integración y continuidad de los esfuerzos de una o más personas, equipos, instituciones comprometidas en el desarrollo del conocimiento en un ámbito específico" (p.5)

Todo este proceso institucional anteriormente descrito se encamina a lograr una producción loable de conocimiento científico, centrada en su función de desarrollo social, como expresión del modo 3 de producción del conocimiento (Acosta Valdeleón \& Carreño Manosalva, s.f.; Jiménez; J. et al. s.f.; Gibbons, M. et al., 1994). Este proceso de gestión social del conocimiento en las IES implica una organización contextualizada para crear riqueza a partir de sus recursos basados en conocimiento, para lo cual se requieren procesos de captura, integración y utilización del Know-How organizacional existente, y las lecciones aprendidas. (Danskin et al., 2005)

Teniendo en cuenta la coincidencia de la estructura organizacional revelada a través del análisis realizado en relación con la revisión teórica realizada, se considera aquí que, hasta el momento, las estructuras organizacionales existentes en las IES responden más a las lecciones aprendidas, entendidas como las experiencias culturales importadas de otros contextos, que a una cultura organizacional propia, emergida a partir del contexto identitario. De la similitud existente entre los sistemas de producción del conocimiento en la investigación institucional, como función sustantiva, en la variedad de IES estudiadas, puede inferirse que han sido objeto de fuertes influencias exógenas, como causa posible para que tantas universidades hayan llegado a construir sistemas de investigación casi idénticos a partir de experiencias disímiles y contextos culturales diversos.

En tal sentido, se reconoce que la historia de la investigación universitaria comenzó por aquellas universidades de Europa como la Universidad de Bolonia y de París, las cuales centraron sus esfuerzos en el desarrollo de la ciencia y por tanto, constituyeron un referente obligado para el desarrollo de la investigación en las universidades que surgieron posteriormente, incluso del modelo universitario alemán, el cual se convirtió en paradigma mundial. De igual modo, la Gran Bretaña en su poderío de metrópoli, expandió múltiples universidades por el mundo, llevando así sus perspectivas de investigación.

Asimismo, Estados Unidos, la ex Unión Soviética y Japón lograron un desarrollo de la ciencia y la investigación universitarias, generando un consecuente incremento de la producción de bienes y servicios a la sociedad, a través de una gestión del conocimiento científico mayormente gestada a partir de sus propios contextos histórico- culturales, sin importar modelos de forma significativa, excepto cuando se referían a procesos de comunicación y comparación de resultados obtenidos. (Sagasti, 1988) 
Este primer estadio de surgimiento de la investigación universitaria ocurrió entonces, de manera endógena, se potenció un alto desarrollo de la llamada industria del conocimiento alrededor no solo de la investigación, sino también de la información y la academia, lo cual evidencia la relevancia del vínculo entre la investigación universitaria y la formación académica.

Siendo así, es evidente que las vías que las universidades de los mencionados países utilizaron para generar investigación científica, necesariamente han influenciado al resto del mundo, pues se convirtieron en modelos paradigmáticos de cómo transitar hacia una producción pertinente de bienes y servicios. Lo anterior es expresión de la lógica del desarrollo cíclico de la cultura universal, en la que aquellos que lograron avanzar de manera temprana se convierten en paradigmas y modelos de los que posteriormente comienzan a surgir.

Sin embargo, es necesario reconocer que no siempre la transferencia de estrategias y modos de producción del conocimiento, garantizan un desarrollo sostenible de la ciencia y la investigación. Por lo general, cada país y universidad en específico necesita reinterpretar aquellos modelos que han dado resultado en otros contextos, desde sus propias experiencias culturales y necesidades sociales, en un proceso de auto-enriquecimiento institucional que ha de conducir a la generación de sus propios enfoques para la investigación científica.

Cada universidad cuenta con un rico potencial interno que le permite generar sus propias revoluciones investigativas institucionales partiendo del nivel de desarrollo teóricopráctico real de sus actores y gestores, del conocimiento empírico acumulado, de las tradiciones y costumbres que han generado la sabiduría popular. Es imposible que universidades que cuentan con alrededor de un cuarto de siglo o menos tiempo de experiencia en la gestión investigativa puedan funcionar con pertinencia con modelos extrapolados de universidades que acumulan siglos de desarrollo, pues los modelos son abstracciones de las realidades y solo funcionan si los sujetos o procesos en los que estos han de funcionar mantienen un nivel de similitud con los contextos que le dieron origen.

Intentar acelerar procesos de desarrollo investigativo universitarios, por medio de la importación e implantación de modelos exógenos, puede ser contraproducente, pues lejos de agilizar el desarrollo, pueden llegar a frenarlo al ser totalmente incomprendidos y disfuncionales, pues no se ajustan a las realidades socio-culturales.

Siendo así, se considera imprescindible potenciar la gestión de investigación como proceso sustantivo universitario a través de modelos endógenos de desarrollo. Dichos modelos deberán generar la construcción de una cultura institucional identitaria, favorecedora de la autonomía intelectual, el pensamiento crítico y la autoctonía profesional del sujeto; en vínculo metodológico con el objeto de la realidad a investigar; lo que direcciona la creación del conocimiento científico y la formación de profesionales, como acción crítica de la propia comunidad universitaria.

Este proceso se constituye en un reto contemporáneo de la Educación Superior, en la sociedad de la información, donde se consumen conocimientos externos -exógenos-, sin una adecuada contextualización. Por ende, se reivindica aquí la necesidad de potenciar la producción de conocimientos desde el interior de la comunidad universitaria -endógenos- que promuevan el perfeccionamiento coherente de investigación como función sustantiva, que aún no ha logrado toda la integración necesaria con la academia y la gestión social del conocimiento para potenciar una formación profesional competente y acorde con la sociedad del conocimiento actual, en las condiciones de globalización. El aporte de la investigación universitaria implica desarrollar una necesaria relación con el entorno social, contribuir a la formación investigativa sostenible de los profesionales por medio de la endogenización del conocimiento científico como elemento de fortalecimiento de la identidad institucional y social.

Este proceso de endogenización del conocimiento puede entenderse desde la propuesta de la Teoría del Pensamiento Complejo de E. Morin (2001). Para él, es una interacción dialógica entre las incertidumbres y expectativas que define la condición humana cognoscente. Ilya Prigogine (2008), por su parte; continuando a Popper, considera el carácter relativo de las certidumbres (conocimiento) y general de las incertidumbres (problemas del conocimiento) como una relación subjetivizada con la realidad del conocimiento que justifica la comprensión de todo sistema cognoscente sólo a partir del caos condicionado por bifurcaciones (o multifurcaciones) para el restablecimiento del orden relativo por alternativas posibles $y$ diversas. 
En tal sentido, Ramírez (2013) ha propuesto un pacto nacional en el Ecuador por la construcción de la sociedad del conocimiento y la creatividad. Para ello, ha marcado acciones que van desde la democratización de la educación superior y del conocimiento; hasta la construcción de un sistema que lo genere, en el marco de una autonomía universitaria responsable y pertinente con la sociedad; que deberá redundar en la endogenización regional del sistema de Educación Superior en América Latina y en el sur global; de este modo se encamina hacia la integración cualitativa de excelencia de las IES y la construcción de una episteme emancipadora, a través de un nuevo régimen académico.

Esta episteme emancipadora -como sistema de integración de cultura colectiva, que logra un crecimiento humano integrador de los sujetos implicados, como protagonistas de su propio desarrollo- articulado con la democracia cognitiva, en la que todos los sujetos se integran armónicamente para la producción de conocimientos socialmente pertinentes, se revela como la tendencia correcta para la construcción de modelos endógenos de investigación científica como función sustantiva universitaria.

Por tanto, en este proceso de creación de bienes y servicios desde el contexto universitario, se hace necesario lograr que el talento humano de las IES genere procesos de aprehensión y apropiación trascendente de la riqueza cultural que la ha antecedido, la preserve, pero no como proceso estático de aislamiento contextual, sino como un proceso sistemático, estructurado y en constante crecimiento desde la interrelación con los presupuestos culturales universales "similares - diferentes, pero igualmente válidos y enriquecedores." (Matos E. \& Cruz R. 2011 p.8).

Este proceso de construcción endógena del conocimiento debe ser capaz de reconocer además, la diversidad de las naturalezas que caracterizan a las ciencias que se investigan. No es posible imponer estructuras y métodos iguales a todas las ciencias y, por tanto, las vías de comunicación científica varían de acuerdo a dicha naturaleza.

La naturaleza de la investigación en Educación Superior contemporánea debe reconocerse como un proceso de abstracción lógica, de carácter complejo y transdisciplinario, interrelacionada con una serie de ciencias y disciplinas particulares (psicología, sociología, antropología, teoría de la comunicación, lingüística, entre otras muchas), lo que demanda una dialéctica de integracióndiferenciación entre ellas, consecuente con lo difuso de las fronteras actuales entre las ciencias, lo que significa la necesidad de investigaciones integradoras $y$ en redes científicas.

Se define así, a la naturaleza de las investigaciones en las IES como la categoría que permite delimitar la existencia del problema científico en el contexto formativo académicoprofesional, así como el objeto y el campo de estudio de dicha investigación dentro de un determinado espacio, tiempo, en relación con otros procesos sociales, ciencias y disciplinas que condiciona, por tanto, la existencia posterior de los resultados científicos y las transformaciones en la realidad institucional, a partir de la participación de los sujetos comprometidos con este proceso. (Matos E. \& Cruz R. 2011)

Se significa aquí entonces, la necesidad de la creación de grupos de intereses científicos de carácter multidisciplinario y transdisciplinario, que permite elevar la competencia colectiva en los procesos de investigación científica en contraposición a la competencia individual, lo que se dinamiza como una cédula organizacional esencial en la contemporaneidad científica. Las redes de conocimiento entonces, son expresiones de procesos de construcción, socialización y transferencia de producción científica motivadas por la globalización y las nuevas tecnologías de la información y la comunicación. Las plataformas tecnológicas fortalecen los procesos de comunicación del conocimiento a nivel internacional y nacional, lo que genera la creación, el intercambio, la cooperación, la potenciación y la transferencia de nuevos conocimientos a nivel social.

En esta dinámica, se reconoce además, la necesidad de la existencia sistematizada de los procesos de divulgación de los resultados investigativos, constituyéndose las publicaciones científicas en una de las vías esenciales para ello. Se reconoce, por tanto, en esta investigación, a las publicaciones científicas como todas aquellas concreciones textuales coherentes con procesos epistémicos comunicativos que evidencian una nueva construcción del conocimiento, a partir de un proceso de sistematización y ruptura investigativa, basadas en métodos, técnicas e instrumentos que demuestran transformaciones cuali-cuantitativas en la naturaleza, la sociedad y el pensamiento 
acorde con la naturaleza de la ciencia en que se investiga.

Se significa, por tanto, que la publicación no existe per se, se constituye en la expresión de un resultado científico y no en un fin en sí mismo. Estas publicaciones científicas, para ser consideradas como tal, deberán ser socializadas en fuentes establecidas y reconocidas por la comunidad científica nacional, regional o internacional como vías para la comunicación de resultados investigativos, dígase revistas indexadas, libros, actas de eventos científicos, informes de investigaciones $\mathrm{u}$ otras posibles.

Es por ello, que se defiende la idea de que todo texto que cumpla con las características anteriormente definidas, se constituye en texto científico, aunque haya sido publicado en una variedad de fuentes, ya sean bases de datos indexadas u otras, también de prestigio regional, internacional, actas de eventos $u$ otras.

Debe tenerse cuidado de no mercantilizar la ciencia. En el mundo contemporáneo, el costo económico de las publicaciones científicas en revistas de alto impacto puede ser elevado, pues ellas se deben a empresas privadas para la comunicación de resultados de ciencia y no todas las universidades cuentan con el potencial económico para solventar ese proceso. No publicar en estas dos bases de datos de alto impacto (Scopus o en la Web of Science) no significa que la producción científica no sea de calidad. Por el contrario, es necesario potenciar el reconocimiento de las bases de datos regionales que son expresión de la cultura del contexto latinoamericano, para prestigiarlas con los mejores resultados científicos alcanzados, de modo que dichas bases, lleguen a mostrar índices de la más alta calidad como manifestación de la producción de conocimientos endógenos.

En tal sentido, es importante resaltar que los resultados de ciencia en la contemporaneidad, en el modo 3 de producción del conocimiento científico, deben ser evaluados más por los impactos alcanzados en el contexto de transformación y por los niveles de satisfacción de los usuarios que reciben dichos impactos que por el ranking de la revista donde apareció la publicación. Lo importante, una vez más, no es la publicación en sí, sino lo que las ideas con ella socializadas significan en términos de crecimiento social para una comunidad universitaria, como expresión de sus propias producciones.

Este análisis ha permitido significar, por tanto, que la Educación Superior contemporánea se determina por las leyes, los principios, las relaciones, las categorías, los métodos, las técnicas e instrumentos científicos que tipifican al proceso de formación del profesional en vínculo con un proceso de gestión social del conocimiento, a partir del complejo sistema de influencias educativas y sociales en el que está inmerso.

Esta definición permite, en un nivel de síntesis, concretar de forma integradora las tres funciones sustantivas universitarias, revelando que una investigación de carácter endógeno, generada desde un proceso de competitividad compartida en redes de conocimiento, configura la formación profesional, de pregrado y postgrado, y la gestión social del conocimiento. Estas funciones, que han sido sustentadas desde el discurso científico como un proceso integrador, aún en la praxis de la Educación Superior necesitan fortalecerse.

No es posible, por tanto, encontrar las vías para desarrollar una integración coherente entre las funciones sustantivas universitarias si no se determina previamente la lógica procesual de la investigación endógena para las IES. Esta lógica procesual está dada en:

- El diseño de la fundamentación epistemológica y metodológica que sustenta la formación del profesional, pre y postgraduado, en relación con el contexto contemporáneo en que se desenvuelve.

- El reconocimiento de la realidad de la cultura científico-académica institucional de los sujetos que conforman la comunidad universitaria.

- La determinación de las tendencias científicas, que con carácter prospectivo, marcan la visión institucional.

- El diseño de planes estratégicos de desarrollo, acorde a la realidad diagnosticada de la cultura institucional científico-académica.

- La determinación de acciones que favorezcan la integración de la investigación formativa con la investigación generativa, que potencie la formación académica y científica de todos los actores institucionales, contribuyendo al desarrollo de una pirámide formativa cíclica.

- La gestión de redes inter-institucionales y el aseguramiento económico para potenciar la participación en eventos de carácter internacional, ya que el fortalecimiento de la investigación también depende del trabajo en red que se realice con las universidades, centros de desarrollo tecnológico, empresas e instituciones del sector público y privado. (Vergara-Quintero, MC., Et. Al., 2016 p.73) 
- La gestión institucional e individual de los investigadores para la transferencia social del conocimiento científico.

- El establecimiento de niveles sistemáticos de publicaciones científicas indexadas, acordes al nivel de desarrollo profesional alcanzado por cada miembro del colectivo universitario docente y a la naturaleza y al estadio del desarrollo de la investigación científica realizada, evitando así el sesgo que ponderan algunas universidades de solo reconocer al artículo científico cuando está publicado en Scopus o en la Web of Science.

\section{Conclusiones}

La Educación Superior en los momentos actuales está abierta a grandes transformaciones en el papel del conocimiento y su gestión, que requieren de procesos educativos de impacto social, con diversidad de alternativas en los procesos formativos, investigativos y de gestión social del conocimiento que, de manera interrelacionada e interdependiente, incorporen a su quehacer científico los múltiples sistemas de influencias sociales para una formación profesional contextualizada. Dicha formación contextualizada ha quedado demostrado que depende, en gran medida, de las interrelaciones funcionales que se establecen entre los procesos que garantizan la existencia universitaria, que no es más que la calidad de la formación integral de sus estudiantes y el crecimiento de la calidad de sus docentes y gestores.

Dichas interrelaciones, implican asumir que la complejidad y urgencia de los problemas de la Educación Superior en la contemporaneidad requieren que la investigación científica contribuya realmente a su solución, desde el espíritu de colaboración en equipos de trabajos dinámicos y transdisciplinares que generen sus propias lógicas de investigación y estructuras teóricas y prácticas a partir de una comunicación que adopte nuevas y cambiantes formas de socialización del conocimiento científico.

Es necesario encontrar un adecuado balance entre la investigación y la responsabilidad social, el acceso a los resultados y los beneficios investigativos acorde a los intereses legítimos individuales y grupales de los contextos de aplicación de la ciencia en un proceso de socialización constante del conocimiento. La investigación, por tanto, debe estar orientada a encontrar su significado social $y$, por ende, sus formas de socialización y comunicación de resultados científicos deberán ser diversos y pertinentes al contexto donde se desarrolla.

Es por ello, que el proceso de endogenización del conocimiento, acorde al desarrollo histórico y contextual de cada una de las universidades, se convierte hoy en día, en una vía imprescindible para el desarrollo identitario institucional de las IES, que con responsabilidad social, están orientadas a encontrar nuevas y diversas alternativas de investigación y de comunicación de sus resultados científicos desde el interior de las instituciones, en un proceso de retroalimentación constante entre lo identitario y lo universal para generar verdaderos procesos de crecimiento endógeno.

\section{Referencias}

Acosta Valdeleón \& Carreño Manosalva Modo 3 de producción de conocimiento: implicaciones para la universidad de hoy. Revista de la Universidad de La Salle. (s.f.).

Barrios, M. Criterios y Estrategias para la definición de líneas de Investigación y prioridades para su desarrollo. Mimeografiado. Caracas: Universidad Pedagógica Experimental Libertador. 1990.

Briceño M, Chapín M. Elementos teóricos para sustentar la evaluación curricular como proceso de transformación y construcción social de las universidades venezolanas. Investigación y Postgrado. Caracas: Universidad Pedagógica Experimental. Disponible en: http://www.scielo.org.ve/ scielo.php?script=sci_arttext\&pid=S1316$00872008000300004 \&$ Ing $=$ es\&nrm=iso\&tlng =es. 2008

Celeiro, A. La cultura ético-axiológica humanista del profesional de la educación desde la formación inicial. Santiago de Cuba: Tesis Doctoral. 2012.

Gibbons, M. et al. La nueva producción del conocimiento. Barcelona: Pomares. 1994.

Holliday J. Orientaciones teórico-prácticas para la sistematización de experiencias. San José, Costa Rica. Disponible en: http://www.bibliotecavirtual.info/wp-content/ uploads/2013/08/Orientaciones_teorico-practicas_para_sistematizar_experiencias.pdf_s/f.

Jiménez; J. et al. Modo 3 de producir conocimiento: investigación socialmente responsable $(2,11,12,14,35)$. Instituto de Investigaciones en Matemáticas Aplicadas y en Sistemas, Universidad Nacional Autónoma de México. s.f. 
Machlup F. The production and distribution of knowledge in the United States, Princeton, Princeton University Press, EUA. 1962.

Matos \& Cruz. La práctica investigativa, una experiencia en la formación doctoral en Ciencia Pedagógica. Ediciones UO, Santiago de Cuba, Cuba. 2011.

Matos \& Cruz. Dinámica epistémicoparticipativa en el proceso de armonización curricular: su construcción praxiológica en la Universidad de Guayaquil, Ecuador. El telégrafo, Guayaquil, Ecuador. 2016

Mintzbery \& Water. Of strategies, deliberate and emergent. En Strategic management Journal. DOI: 10.1002/smj.4250060306. 1985.

Morin, E. Los Siete Saberes necesarios para la Educación del Futuro. Editorial Magisterio con licencia de UNESCO. Bogotá. 2001.

Prigogine, I. Las leyes del caos. Editorial Crítica-Drakontos Bolsillos, Madrid, España. 2008.
Ramírez R. Tercera Ola de Transformación de la Educación Superior en Ecuador. Secretaría Nacional de Educación Superior, Ciencias Tecnología e Innovación, Ecuador. 2013.

Sagasti F. R. Conocimiento y desarrollo: ensayos sobre ciencia y tecnología. Mosca Azul, Lima, Perú. 1988.

Velázquez Méndez A. \& Maldonado Talamantes La especificación de procesos en las instituciones públicas de educación superior. En: Teoría y Praxis. Disponible en: https://dialnet.unirioja.es/servlet/articulo?codi go $=2929741.2006$.

Vergara-Quintero, MC., Et. Al., Teoría de recursos y capacidades para evaluar la función sustantiva de investigación en instituciones de educación superior. Revista educación y desarrollo social. 10(1), 58-77. DOI: $\quad$ http://dx.doi.org/10.18359/reds.1448. 2016. 